\section{Surveying Black or African American Populations: Challenges and Solutions in Medicine and Education}

\author{
Kenneth D. Royal \\ Department of Clinical Sciences, North Carolina State University, \\ $U S A$
}

To cite this article: Royal KD. Surveying Black or African American populations: challenges and solutions in medicine and education. Education in Medicine Journal. 2019;11(2):59-61. https://doi. org/10.21315/eimj2019.11.2.8

To link to this article: https://doi.org/10.21315/eimj2019.11.2.8
Kenneth Royal, PhD, Department of Clinical Sciences, North Carolina State University, 1060 William Moore Dr., Raleigh, NC 27607, USA | E-mail: kdroyal2@ncsu.edu
Research in the fields of public opinion, demography and sociology, health and medicine, and education have long noted disproportionately lower survey response rates from Black or African American populations compared to other population groups (1-4). Numerous factors have been attributed to these lower participation rates. For example, logistical issues, participant unwillingness, differing attitudes about social responsibility and poor reading skills have been reported for mail surveys across multiple contexts (5). In the context of health-related surveys, a myriad of complex factors affects participation rates. Dating back to the Tuskegee syphilis study and continuing today with modern health disparities, many Black or African Americans remain distrustful of researchers (6). Further, lack of understanding, suspicion of purpose, fear of how information will be used, inaccessible language (e.g., medical jargon, technical terms) and beliefs that Black or African Americans have been overly researched have also been reported (7-8).

In the context of higher education surveys, Black or African American students have been reported to provide response rates approximately $20 \%-25 \%$ lower than other racial and ethnic groups (9). Reasoning includes feelings of disenfranchisement and skepticism that their feedback will be taken seriously (10). In other contexts, factors such as disengagement from government institutions and lack of ability to comprehend survey items typically developed by persons who are members of the racial/ethnic majority and middle-class socioeconomic groups have been reported $(7,11)$.

Low participation rates for Black or African American populations pose a considerable problem for any survey research effort. Low participation rates typically result in non-representative data, a major validity threat that both skews overall results and may distort the inferences researchers make about the findings. In the context of large-scale surveys, researchers often have the ability to perform techniques such as stratified random sampling or quota sampling to offset any initial sampling deficiencies relating to a particular subpopulation or group. In other contexts, in which it may not be possible to survey additional individuals' researchers may need to utilise post-stratification weights (12) to statistically adjust for under/overrepresented groups. Unfortunately, however, 
most survey researchers have limited budgets and limited access to potential participants, so repeated waves of survey invitations are not viable. Further, survey researchers often have limited proficiency in the tedious and technical process of generating statistical weights to correct for sampling bias. Thus, it is critical that survey researchers are able to capture the best quality data possible during the first wave of data collection.

Fortunately, a few potential solutions have been proposed for increasing Black or African American participation rates. Some researchers have noted personal visits, when feasible, may help (13). The literature on incentives has noted the use of immediate and unconditional monetary incentives have been evidenced to increase response rates among virtually all racial and ethnic groups (14). For survey researchers with a limited budget and/or conducting studies outside a community in which personal contact is an option, there are a few general approaches that have been demonstrated to be successful.

Perhaps the most significant strategy involves communication. Clear communication, which includes clearly articulating what the research is about, why it is being conducted, why it is important to participants and their communities, and emphasising the value of the participant's perspective is critical for building trust and legitimacy (8). Other forms of communication, such as simplifying the wording of items and utilising examples and images that include Black or African American persons can help demonstrate cultural awareness $(10,15)$. Where possible, it may be helpful to "close the loop" by sharing survey findings with participants and noting what specific changes were made from participants' feedback. In sum, the best strategies for improving Black or African American response rates involve honesty, respect, sincerity and clear communication; the same qualities that should underpin all survey research efforts.

\section{REFERENCES}

1. Fan W, Zheng Y. Factors affecting response rates of the web survey: a systematic review. Comput Human Behav. 2010;26(2):132-9. https://doi.org/10.1016/j.chb.2009.10.015

2. Dillman D, Sinclair M, Clark J. Effects of questionnaire length, respondentfriendly design, and a difficult question on response rates for occupant-addressed census mail surveys. Public Opinion Quarterly. 2003;57(3):289-304. https://doi. org/10.1086/269376

3. Herring $P$, Butler $T$, Hall $S$, Bennett $H$, Montgomery SB, Fraser G. Recruiting and motivating Black subjects to complete a lengthy survey in a large cohort study: an exploration of different strategies. BMC Med Res Method. 2014;14(1). https://doi. org/10.1186/1471-2288-14-46

4. Bonevski B, Randell M, Paul C, Chapman K, Twyman L, Bryant J, Brozek I, Hughes C. Reaching the hard-to-reach: a systematic review of strategies for improving health and medical research with socially disadvantaged groups. BMC Med Res Method. 2014;14(1):42. https://doi. org/10.1186/1471-2288-14-42

5. Bowling A. Mode of questionnaire administration can have serious effects on data quality. J Pub Health. 2005;27(3):28191. https://doi.org/10.1093/pubmed/fdi031

6. Scharff DP, Mathews KJ, Jackson P, Hoffsuemmer J, Martin E, Edwards D. More than Tuskegee: understanding mistrust about research participation. J Health Care Poor Underserv. 2010;21(3):879-97. https://doi.org/10.1353/ hpu. 0.0323

7. Smith P. Review of BME coverage in the healthcare commission's patient survey programme. 2006 [cited 24 March 2019]. Available from: http://www.nhssurveys.org/ Filestore/documents/Review_BMEcoverage_ HCC_surveys.pdf. 
8. Elam G, McMunn A, Nazroo J. Feasibility study for health surveys among black African people living in England. Final report implications for the Health Survey for England 2003. Joint Health Surveys Unit of University College London and the National Centre for Social Research; 2001 [cited 24 March 2019]. Available from: http:// discovery.ucl.ac.uk/175921/

9. Krebs $\mathrm{CP}$, Lindquist $\mathrm{CH}$, Barrick $\mathrm{K}$. The historically Black college and university campus sexual assault (HBCU-CSA) study. PsycEXTRA Dataset, 2010. https://doi. org/10.1037/e545562011-001

10. Royal KD, Singletary GT. Black student participation rates also a concern for educational program assessments. J Nat Black Nurses Assoc. 2017;28(2):40-3.

11. Owens L, Johnson TP, O'Rourke D. Culture and item nonresponse in health surveys. In: Cynamon M, Kulka RA. Seventh conference on health survey research methods. Proceedings. Hyattsville, MD: U.S. Dept. of Health and Human Services, Public Health Service, Centers for Disease Control and Prevention, National Center for Health Statistics; 2001. https:// doi.org/10.3886/icpsr29022
12. Royal KD. Survey research methods: a guide for creating post-stratification weights to correct for sample bias. Educ Health Prof. 2019;2(1).

13. Griffin DH. Measuring survey nonresponse by race and ethnicity. United State Bureau of the Census: Washington, DC, 2002 [cited 24 March 2019]. Available from: https:// www.census.gov/content/dam/Census/ library/working-papers/2002/acs/2002_ Griffin_01.pdf.

14. Beebe TJ, Davern ME, Mcalpine DD, Thiede-Call K, Rockwood TH. Increasing response rates in a survey of medicaid enrollees. Med Care. 2005;43(4):411$14 . \quad$ https://doi.org/10.1097/01. mlr.0000156858.81146.0e

15. National Center for Education Statistics. The health literacy of America's adults: results from the 2003 national assessment of adult literacy. Washington, DC: U.S. Department of Education; 2006. 